\title{
Ossification of the lumbosacral dura and arachnoid following spinal cord trauma. Case report
}

\author{
RB Bell ${ }^{1}$, CJ Wallace ${ }^{2}$, HA Swanson ${ }^{1-3}$ and AKW Brownell ${ }^{1}$ \\ Departments of ${ }^{1}$ Clinical Neurosciences and ${ }^{2}$ Radiology, Foothills Hospital, University of Calgary; ${ }^{3}$ Department \\ of Diagnostic Imaging, Calgary General Hospital, Calgary, Alberta, Canada
}

\begin{abstract}
A case of progressive cauda equina syndrome secondary to ossification of the lumbosacral dura arachnoid following an incomplete spinal cord injury is presented. The etiology of the ossification is unclear and may be secondary to heterotopic osseous metaplasia such as occurs in other sites in spinal cord injured patients.
\end{abstract}

Keywords: heterotopic ossification; intraspinal ossification; spinal cord injury; osseous metaplasia

\section{Introduction}

Intradural ossification secondary to spinal cord injury has been infrequently described. ${ }^{1}$ The best documentation of this phenomenon comes from a series of autopsy and cordotomy specimens, representing sequalae of spinal cord injury producing paraplegia, and which were largely incidental findings at the time of pathological examination. ${ }^{2}$ The mechanism of ectopic ossification within the dural sac may be ascribed to intradural hematoma, the seeding of bone fragments from traumatic injury or spinal surgery, or to heterotopic osseous metaplasia. The latter is a well recognized complication of spinal cord injury, affecting $16-53 \%$ of patients. ${ }^{3}$ The incidence appears to be higher in those with complete neurological injuries. Almost invariably these lesions develop below the site of neurological injury and usually occur within muscle and periarticular soft tissues. Frequently they are accompanied by a local inflammatory reaction and fever. The underlying pathophysiology of this disorder has not been clarified. ${ }^{4-7}$

We present a case report of a progressive cauda equina syndrome, developing following incomplete spinal injury and presumed secondary to ossification within the lumbar dura arachnoid. Of the potential mechanisms for this ossification we suggest that heterotopic ossification in an unusual site is the likely cause.

\section{Case report}

This 40-year-old male was initially evaluated at the University of Calgary neuromuscular clinic in 1986. In 1969, at the age of 22 , he had been involved in an airplane crash and sustained a compression fracture with fragmentation of the second lumbar vertebral body resulting in paraparesis. A

Correspondence: RB Bell, Department of Clinical Neurosciences, University of Calgary, Health Sciences Centre, 3330 Hospital Drive NW, Calgary, Alberta, Canada, T2N 4N1. fusion extending from T11 to L3 vertebral bodies was performed approximately 3 months following the injury. He recovered gradually, regaining almost complete motor function, and with no sensory or sphincter disturbance. He has had constant pain at the surgical/injury site since the time of the accident and by 1984 he had developed pain radiating into his right buttock and right posterior leg. In addition, he developed sensory symptoms in his sacral dermatomes and urinary sphincter disturbance, with dribbling incontinence. He denied progressive motor weakness.

On examination he had percussion tenderness over his upper lumbar spine and a moderate lower thoracic kyphosis. Muscle atrophy was present in both the calf and the peroneal muscles and he had prominent fasciculations in both thighs. Strength was mildly diminished in the proximal right leg and hip, tone was normal and tendon reflexes were absent in both legs. A sensory level, without sacral sparing, was present below lumbar 2 . Rectal sphincter tone was diminished and his anal wink was absent.

A clinical diagnosis of a diffuse and progressive denervating process below L2, involving both anterior and posterior roots within the cauda equina and affecting the conus-epiconus region, was made.

Radiographic evidence of calcification was first identified in 1982 by plain films, which showed a linear calcific density within the spinal canal from approximately L3 to S1 vertebral bodies (Figure 1). Computerized axial tomography $(\mathrm{CT})$, at that time, revealed an enlarged subarachnoid space at the injury site which extended over one vertebral level distal to the fracture, as well as extensive calcification of the distal dural tube. A repeat CT scan in 1986 once again demonstrated the calcific change of the dural sac extending from L3 to the sacral hiatus (Figure 2), which had become more extensive when compared with the first scan done in 1982.

The patient was seen again for review in 1993. At that time the patient complained of a further deterioration in the strength in his lower extremities. He continued to have urinary sphincter disturbance and he complained of a constant burning pain in his legs and perineum. On examination, he had considerable atrophy of his right quadriceps and peroneal muscles, as well as the medial aspect of his right calf. Fasciculations were present in most muscle 


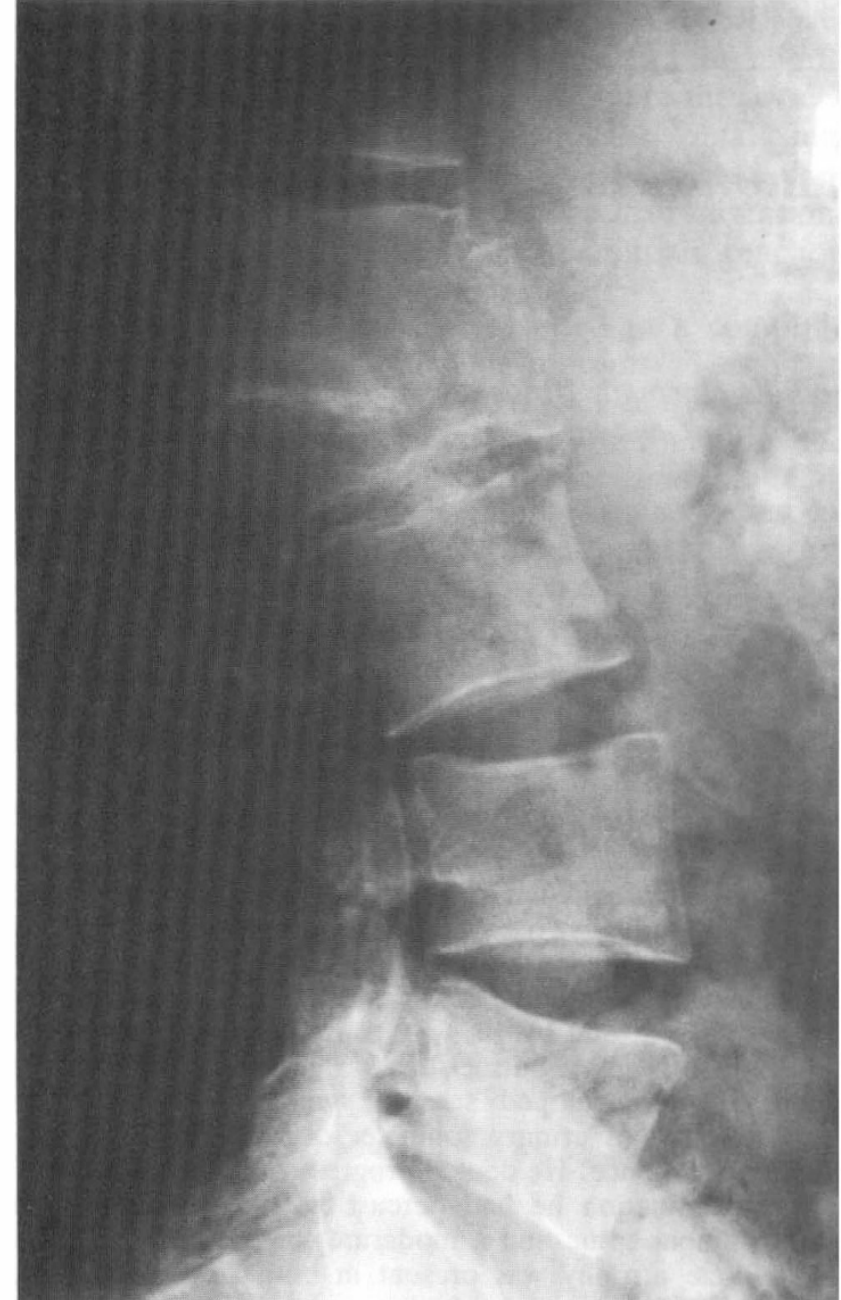

Figure 1 Lateral lumbar spine: L2 has been impacted and fused with the body of L1. A calcific density is seen within the spinal canal below this level and extending to the sacral region

groups in his legs and tone was normal. He had mild weakness of hip flexors bilaterally and of his right quadriceps and hamstrings but he maintained normal power in the hip abductors, adductors and extensors. There was a fused left ankle, but he maintained extension and flexion of his toes. There was moderate weakness of right ankle dorsiflexion and plantarflexion. Sensory examination revealed a sensory level to pinprick and light touch at L2 bilaterally. He had loss of vibration sensation to the level of his knees, loss of joint position on the right to his hip and on the left to his ankle.

An MRI scan was performed in 1990 and repeated in 1993 (Figure 3). This revealed an old burst fracture of L2 with obliteration of the L1-2 disk space and healed fracture fragments from L2 encasing the L1 vertebral body. There was a mild angular deformity and evidence of a prior decompressive laminectomy. A large pseudomeningocele was present extending from the T12/L1 to the L2-3 interspace. The conus was seen within this region. There was evidence of calcification encasing the dural sac inferior to the pseudomeningocele and extending distally to the sacral hiatus. This remained unchanged at the time of the 1993

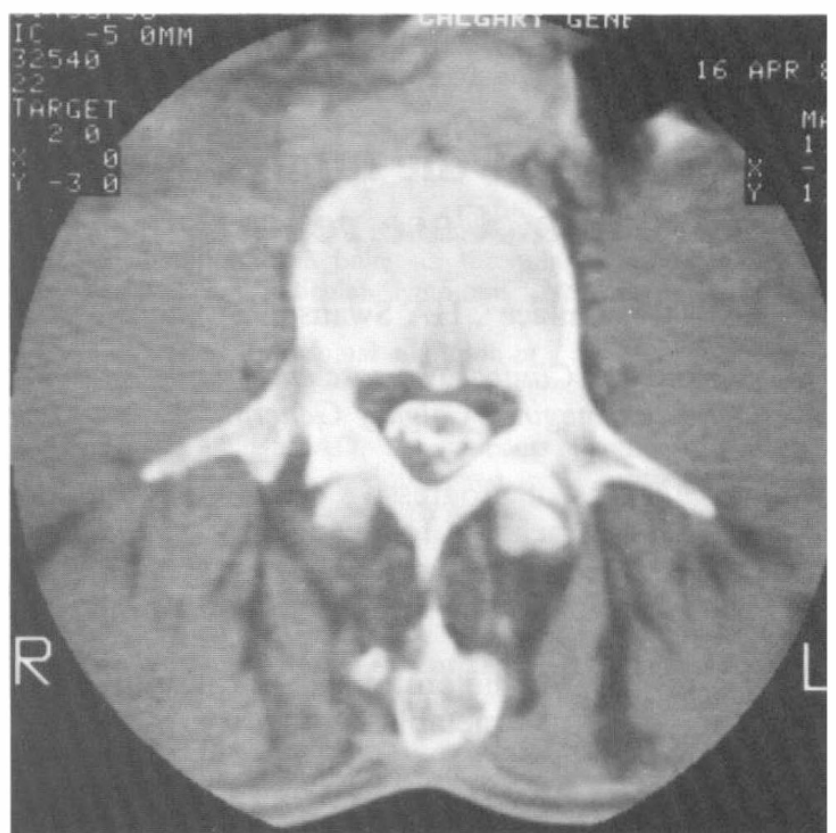

b

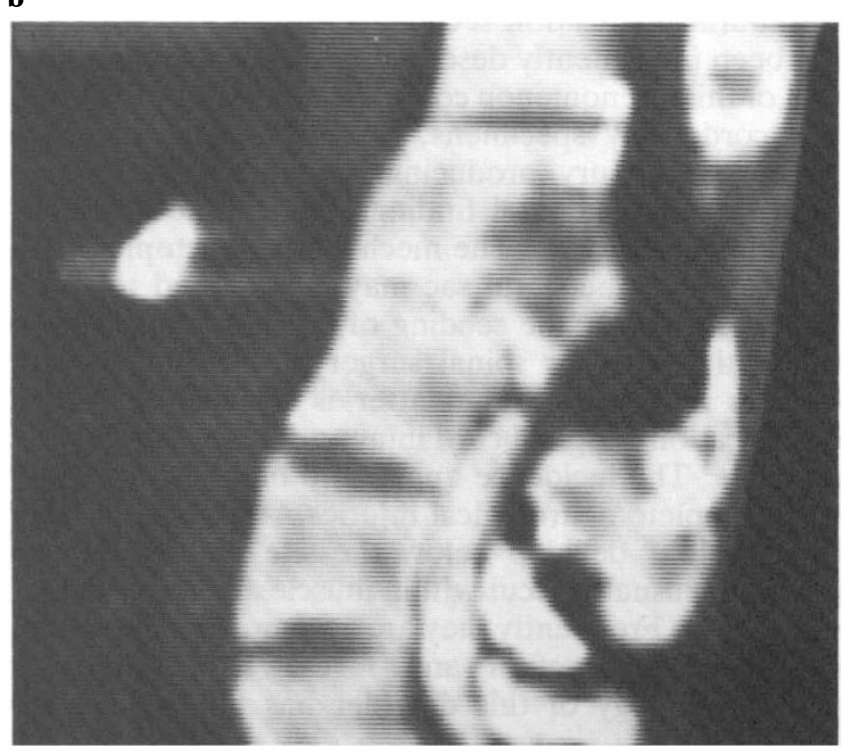

Figure 2 (a) Axial non-contrast CT scan of lumbar spine: L4 level demonstrating dural and intradural calcification. (b) Sagittal CT scan (reconstructed) of lumbar spine demonstrating calcified dural tube

MRI (Figure 3). The patient continues to be followed up by the authors and the indolent progression of his neurological deficit has continued, albeit slowly. The ultimate prognosis remains uncertain.

\section{Discussion}

The complex nature of the pathology that is present limits any definitive statements regarding the etiology of the patient's progressive neurological syndrome. Although the presence of a meningocele or pseudo- 


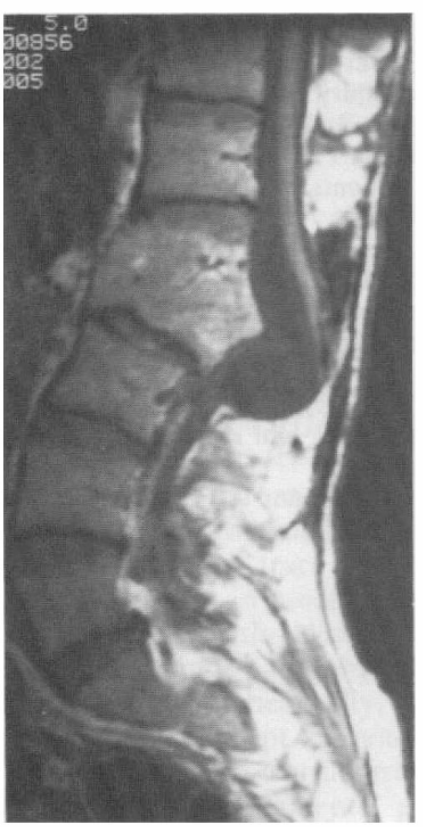

b

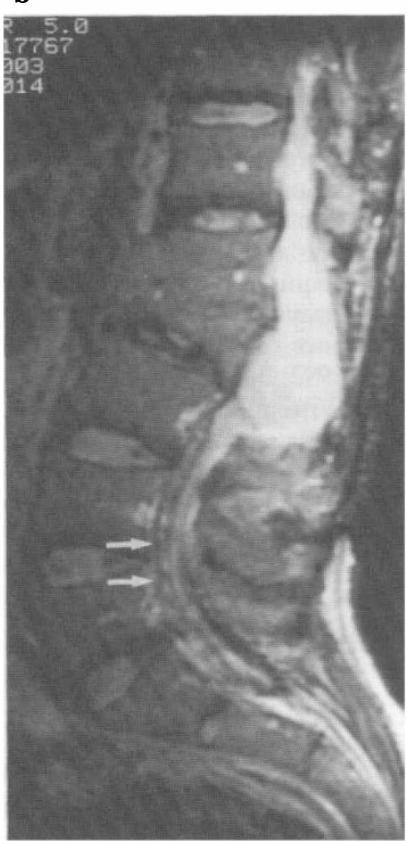

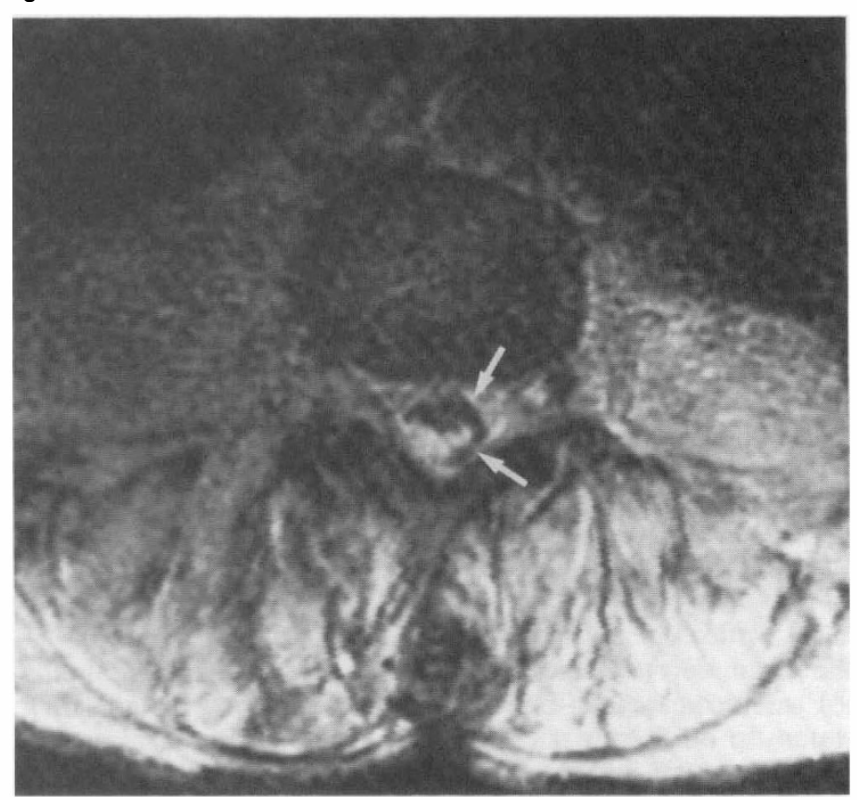

Figure 3 MRI of the lumbosacral spine. (a) Sagittal T1-weighted image (TR 650, TE 19). (b) Sagittal T2-weighted image (TR 2000, TE 70). (c) Axial T2*-weighted gradient echo image (TR 40, TE 15, theta $5^{\circ}$ ). An old healed fracture of L2 with obliteration of the L1-2 disc space, angular kyphotic deformity and post-traumatic pseudomeningocele (with the conus lying posteriorly). Marked hypointensity due to calcification (arrows) is present along the anterior and left lateral aspect of the dural sac below the L2-3 level on the T2-weighted images with only small amounts of normal bright CSF visible on the right posterolaterally

meningocele resultant from the trauma incurred may be implicated in permanent neurological deficit manifested from the time of injury, it does not adequately explain the longstanding indolent progression of the neurological compromise seen in this patient. Imaging studies demonstrate no impingement on the conus and epiconus although some nerve root stretching may result from posterior displacement of the lower spinal cord. The nature of the pseudomeningocele has not changed substantially since the first CT scans were done in 1982. The bony injury and subsequent bony fusion from T11 to L3 vertebral bodies has resulted in expansion of the right first and second lumbar nerve root foramina and we have no radiological evidence to support the hypothesis that bony overgrowth has resulted in foraminal impingement. The clinical course and radiographic imaging studies lead us to believe that the progressive neurological deficit evident in this case is the result of dural and intradural ossification producing traction or direct pressure on nerve roots and the lower spinal segments.

Several mechanisms, all hypothetical, may be postulated to explain the etiology of the ossification process. An intradural hematoma arising at the time of the initial injury may have incompletely resorbed and subsequently organized, leading to subsequent calcification and ossification. The extent of calcific change would imply a significant hemorrhagic event intradurally and, considering the limited previous extent of neurological deficit, we felt that this was an unlikely event. It is possible that the intradural space was seeded by bone fragments arising from the injury or the surgical intervention and acted as a nidus for more extensive calcification. No discrete bone fragments were evident radiologically however and, despite the frequency of complicated spinal fractures and remedial surgical procedures, intradural ossification has rarely been described. Calcification of a low grade inflammatory process would imply an indolent infective process. It is notable that the patient was being treated with isoniazid because of a pulmonary nodule that had been noted on a routine chest radiograph and his tuberculin test was found to be positive when he was first evaluated by the authors in 1986; however this was several years after the calcification was first noted and the patient would be expected to have had a greater degree of clinical disease if chronic tuberculosis was responsible. In addition, we have not demonstrated any central nervous system focus of infection.

Heterotopic ossification occurs frequently in patients with spinal cord injury. The usual sites of involvement are the proximal joints and limbs and the presence of spasticity may partially direct site specificity. ${ }^{6}$ The indolent course of this patient's neurological deficit and the absence of evidence supportive of an alternative mechanism suggest that intraspinal dural-arachnoid heterotopic osseous metaplasia may be responsible. The etiology of this phenomenon remains obscure ${ }^{4,5,7}$ 
Apart from the spinal fusion, no surgical or pharmacological intervention has been made. Disodium etidronate was not used because of the presence of extensive intraspinal dural-arachnoid ossification at the time of presentation, the predisposition to bony fractures with prolonged use of this agent, and the risk of rebound ossification upon discontinuation. ${ }^{8}$ Radiological evaluation in this case has elucidated the mechanism underlying the development of the progressive neurological deficit, but does not assist in understanding its pathogenesis.

\section{References}

1 Kinnunen J, Laasonen EM. Posttraumatic lumbar intraspinal extradural-intradural ossification. Neuroradiology 1990; 32:
$160-162$.

2 Hardy AG, Dickson JW. Pathological ossification in traumatic paraplegia. J Bone Joint Surg Br 1963; 45: 76-87.

3 Finerman GAM, Stover SL. Heterotopic ossification following hip replacement or spinal cord injury: two clinical studies with EHDP. Metab Bone Rel Res 1981; 3: 337-342.

4 Kurer MH, Khoker MA, Dandona P. Human osteoblast stimulation by sera from paraplegic patients with heterotopic ossification. Paraplegia 1992; 30: 165-168.

5 Garland DE. A clinical perspective on common forms of acquired heterotopic ossification. Clin Orthop 1991; 263: 13-29.

6 Bravo-Payno $\mathrm{P}$ et al. Incidence and risk factors in the appearance of heterotopic ossification in spinal cord injury. Paraplegia 1992; 30: 740-745.

7 Wittenberg RH, Peschke U, Botel U. Heterotopic ossification after spinal cord injury. Epidemiology and risk factors. $J$ Bone Joint Surg Br 1992; 74: 215-218.

8 Buschbacher R. Heterotopic ossification: A review. Crit Rev Phys Rehabil Med 1992; 4: 199-213. 